\title{
Long-term treadmill exercise upregulated hippocampal learning-related genes without improving cognitive behaviour in socially isolated rats
}

\author{
S. Keloglan', L. Sahin', O.S. Cevik ${ }^{2}$ \\ ${ }^{1}$ Department of Nursing, Faculty of Health Sciences, Amasya University, Amasya, Turkey \\ 2Department of Physiology, Faculty of Medicine, Mersin University, Mersin, Turkey \\ [Received: 30 November 2018; Accepted: 31 January 2019]
}

Background: Some environment enrichments such as exercise has been reported to improve the diminished cognitive functions and related gene expression. Therefore, we aimed to investigate the effects of prolonged treadmill exercise on long-term learning and hippocampal gene expression, which involves learning and plasticity.

Materials and methods: Male Wistar rats $(n=32)$ randomly assigned into four groups: control (C), social isolation (SI), exercised (E), social isolation + exercise (SE) during postnatal days (PNDs) 21-34. Social isolation protocol was applied during 14 days by placing the rat alone in a cage. Rats were exercised daily, 5 days per week, for overall 4 weeks. Finally, learning performance was evaluated by the novel object recognition test. At the end of learning test, the rats were decapitated to isolate hippocampus tissues for learning related gene expression such as N-methyl-d-aspartate receptor (NMDAR) subunit genes (Grin1, Grin2a, Grin2b) and cyclin dependent kinase 5 (Cdk5), Cdk5 regulatory subunit p35 (Cdk5r), activity-regulated, cytoskeletal-associated protein (Arc), the immediate early gene (c-Fos, a marker of neuronal activation), doublecortin (DCX), achaetescute homolog 1 (ASCL1), brain-derived neurotrophic factor (BDNF) by real-time polymerase chain reaction (RT-PCR).

Results: Grin1, NMDAR subunit gene expression was increased significantly in E group compared to other groups. Grin2b, NMDAR subunit gene expression was increased in E group compared to the SI group. Cdk5 level increased in E group compared to the SE group. The ASCL1 gene expression increased in E group compare to the SE group. The DCX gene expression increasing in C group compared to SI and SE groups.

Conclusions: Taken together these findings may point out that long-term social isolation down-regulated learning-related genes. However, treadmill exercise together with social isolation did not restore this down-regulation although treadmill exercise increased learning-related genes without improving cognitive behaviour. (Folia Morphol 2019; 78, 4: 668-675)

Key words: learning, neurogenesis, NMDAR, plasticity, BDNF, novel object test 


\section{INTRODUCTION}

Increasing evidence has shown that environmental disruptions such as social isolation stress can cause severe cognitive dysfunctions [15]. Cognitive functions like learning and memory occur in the hippocampus and it's developmental stages affected by stress factors in the adolescent period [40, 46]. Additionally, experiencing social isolation stress in adolescent period caused anxiety and depression-like behavioural changes and impaired cognitive functions in the adult lifetime $[23,27,38,39]$. The benefits of exercise are also apparent in the central nervous system where exercise can protect against neurodegenerative disorders, alleviate symptoms of psychiatric disorders such as depression and anxiety or facilitate learning and memory $[2,10,20,24,26]$. Learning and memory occur in hippocampus particularly dependent on the hippocampal circuit and involves induction of some N-methyl-d-aspartate receptor (NMDAR) subunit genes. Glutamergic system and NMDA-mediated glutamate system are known to be crucial for brain development, neuronal migration, differentiation and hippocampal plasticity [49].

NMDAR contains a heterotetrameric complex of two Grin1 subunits and two Grin2 subunits (Grin2A-2D). Grin1 gene produces GluN1 protein and essential for the formation of functioning NMDARs [32]. In addition, several mutations in Grin1 caused severe developmental delay associated with intellectual disability [33]. NMDAR subunits Grin2a and Grin2b genes are highly abundant in brain regions associated with memory functions and hippocampal plasticity [11]. Also, Arc is important for maintenance of long-term plasticity and memory $[35,36]$.

One important feature of hippocampal plasticity that might make it sensitive to the stress, and also differentiates it from most other brain regions, is the fact that the hippocampus still comprises stem cells that increase new neurons even in adulthood. For instance, NMDAR activity is necessary for the regulation and correction of existing neuronal circuits during the acquisition of new information, the maturation of dendritic spines, synaptic connections and excess activation can cause excitotoxicity [28, 45]. NMDAR also appear in the calcium-dependent brain-derived neurotrophic factor (BDNF) stimulation pathway and required for activity-dependent BDNF expression and BDNF suppression [8, 44]. BDNF is the vital neurotrophic factors that links exercise with improvements in cognitive processes [4]. Also, the
Cdk5/p35 pathway has been known closely related to NR2B in spatial learning and memory functions $[30,37]$. Cdk5 is known to phosphorylate doublecortin (DCX) and other microtubule-regulatory proteins [9]. DCX located in the granular layer of the dentate gyrus (DG) in the hippocampus has shown to be critical in the development via mitosis of neural stem cell [17]. Also DCX involves in migrating neuroblasts and regulates neuronal migration by stabilising of microtubule function [16]. Achaete-scute homolog 1 (ASCL1) plays a central role in the differentiation of neuronal progenitor which is expressed by hippocampal progenitors during development [34]. In addition, Fos proteins which product of c-Fos gene involves in cell growth, proliferation, differentiation [16, 25, 41].

The aim of the present study was to determine the effect of exercise on learning and memory functions by using novel object recognition test, and the expression of hippocampal genes, namely NMDAR (Grin1, Grin2a, Grin2b), BDNF, Cdk5, Cdk5r, c-Fos, DCX, Arc, ASCL1 was measured by real-time polymerase chain reaction (RT-PCR) in the socially isolated rats.

\section{MATERIALS AND METHODS}

Animals and experimental groups

Experimental procedures were performed in accordance with the Mersin University of Health Guide for Care and Use of Laboratory Animals and were approved by the University of Mersin Institutional Animal Care and Use Committee. Male Wistar rats $(n=32)$ 3 weeks old, exposed to an inverse 12-h light/dark cycle at ambient temperature of $21 \pm 1^{\circ} \mathrm{C}$. After the acclimatisation period, male rats were randomly divided into four groups $(n=8)$ : control $(C)$, social isolation stress $(\mathrm{SI})$, exercise $(\mathrm{E})$, social isolation stress + exercise (SE).

\section{Social isolation procedure}

Social isolation involved removing the experimental animal from the home cage, and placing it into an isolated cage with dimensions $25 \times 42 \times 15 \mathrm{~cm}$. Every day rats were exposed to stress between 08:00 and 14:00 for $6 \mathrm{~h}$ in a separate room. After completed their social isolation, rats spent the rest of the day in standard rat cages. This procedure was repeated daily for 14 days. During this period, the control groups $(C, E)$ spent their time in the standard rat cages [6].

\section{Exercise procedure}

After 14 isolated days, for physical exercise procedure, the treadmill apparatus was used. The running 
motivation of the animals was enhanced by direct current shock $(0.1-0.15 \mathrm{~mA})$ at the lane terminal of the treadmill [5]. Rats were exercised daily, 5 days per week, for overall 4 weeks. Everyday exercise procedure began 14:00, between $08: 00$ and 14:00 social isolation protocol was continued (SI, SE). The running time in exercise groups was increased progressively through 4 weeks. The first-week duration of exercise was $20 \mathrm{~min}$, second week - $50 \mathrm{~min}$, third week - $50 \mathrm{~min}$ and last week $60 \mathrm{~min}$. After exercise protocol learning performances were evaluated by novel object recognition test.

\section{The novel object recognition test}

Distinct visual cues were placed on the walls of the testing room and a video camera was positioned overhead to record animals' interactions with the objects during both the familiarisation and test phase $[14,29]$. Before began the test rats were familiarised to the area for $15 \mathrm{~min}$ and rats underwent Spatial Object Location test sessions the next day. Each test session consisted of a 3-min familiarisation phase and a 3-min test phase, which were separated by a delay ( $60 \mathrm{~min}, 15 \mathrm{~min}$, and $240 \mathrm{~min}$, respectively). The first day, after 3-min familiarisation phase, 3-min test phase was applied after $60 \mathrm{~min}$ delayed. Second day delay time determined as $15 \mathrm{~min}$ and third day it was $\mathbf{2 4 0} \mathrm{min}$. Two identical objects were located in the corners of the arena furthest from the experimenter and the time spent investigating each object was measured. A rat was considered to be exploring an object when it was directing its nose at a distance of less than $2.5 \mathrm{~cm}$ to the object and/ /or touching it with its nose [14]. After the delay that followed familiarisation, rats underwent a 3-min test phase. During the test phase, rats were returned to the same arena, except that one of the two objects seen before was moved to one of the previously unoccupied corners. The object that was moved and the corner that it was moved to were counterbalanced across condition and test sessions, such that rats never experienced the same object relocation twice. The amount of time that animals spent investigating each object was measured. Because rats have an innate preference for novelty, if their spatial memory is intact they will spend more time with the object in the new location compared to the object that was not moved [18].

At the end of all experiment, under the ketamine/ /xylazine anaesthesia, rats were decapitated and their hippocampus tissue was isolated. The brains were stored at $-80^{\circ} \mathrm{C}$ and used for gene analysis with the method of the quantitative RT-PCR.

\section{Gene expression by RT-PCR and analysis}

Total RNA isolation from the tissues was performed by a manual method using TRIzol (Invitrogen). $4 \mu \mathrm{L}$ total RNA extraction was used as a template into synthesis of CDNA using Fluidigm Reverse Transcription Kit in accordance with the manufacturer's instructions to obtain CDNA. Quantitative RT-PCR was run on a Fluidigm Biomark Real-Time PCR using Taqman GE Master Mix. The Fluidigm Access Array (TaqMan) was used to evaluate expression levels of Grin1, Grin2a, Grin2b, Cdk5, Cdk5r, Arc, c-Fos, DCX, ASCL1, BDNF. The relative expression of genes was calculated by the comparative $2^{-\Delta \triangle c t}$ method using PPIA RNA levels as internal control. Peptidylprolyl widely preferred as a control gene in neurological research [19].

\section{Data evaluation and statistical analysis}

All data are expressed as mean values \pm standard error of the mean (SEM). The normal distribution of data in each group was controlled by the Shapiro-Wilk test. For normally distributed data of behavioural test and genes expression level, statistical analysis was performed by using one-way ANOVA, repeated measures analysis of variance (ANOVA) and Tukey post hoc comparisons. The significance level of $p<0.05$ was considered for statistically significance.

\section{RESULTS}

Effects of social isolation and exercise on novel object recognition test at different time intervals

The time spent in the familiar object was evaluated in all intervals after the training session. As a normal behaviour, rodents usually spent more time exploring the novel object in the test session, which indicates that they recognised the object offered previously. According to the novel object recognition test, there is a significant difference at a 60 -min section in social isolation group compare to the control ( $p<0.05$ ). For the group of rats that performed test session 15 and 240 min after training, all groups recognised similarly the novel object, since all groups of rat spent the same time in the novel object in the test (Table 1).

\section{Effects of social isolation and exercise on hippocampal learning related and neurogenesis gene levels}

According to our results; Grin1 NMDAR subunit gene expression was increased significantly in $\mathrm{E}$ group compared to other groups (for $C$ group, $p=0.049$; for $S I$ group, $p=0.001$; for $S E$ group, $p=0.000$ ). Grin $2 b$ 
Table 1. Exploring time for novel object test

\begin{tabular}{lccc}
\hline Groups & \multicolumn{3}{c}{ Time/minute } \\
\cline { 2 - 4 } & $\mathbf{1 5}$ & $\mathbf{6 0}$ & $\mathbf{2 4 0}$ \\
\hline $\mathrm{C}$ & $3.57 \pm 2$ & $92.50 \pm 39^{*}$ & $40.83 \pm 30$ \\
SI & $34.38 \pm 21$ & $18.54 \pm 6$ & $41.25 \pm 18$ \\
CE & $14.18 \pm 6$ & $67.91 \pm 19$ & $62.29 \pm 24$ \\
SE & $29.8 \pm 13$ & $74.79 \pm 13$ & $85.83 \pm 40$
\end{tabular}

${ }^{*}$ There is a significant difference at the 60 -min section in social isolation group compare to the control $(\mathrm{p}<0.05)$. Values are given as mean \pm standard deviation $\mathrm{C}$ - control group; $\mathrm{SI}$ - social isolation group; $\mathrm{E}$ - exercised group; $\mathrm{SE}$ - social isolation + exercise group

NMDAR subunit gene expression was increased in E group compared to SI group (for SI group, $p=0.012$ and for SE group, $p=0.008)$. In contrast, Grin2a gene expression did not change between groups $(p=0.81$; Fig. 1).
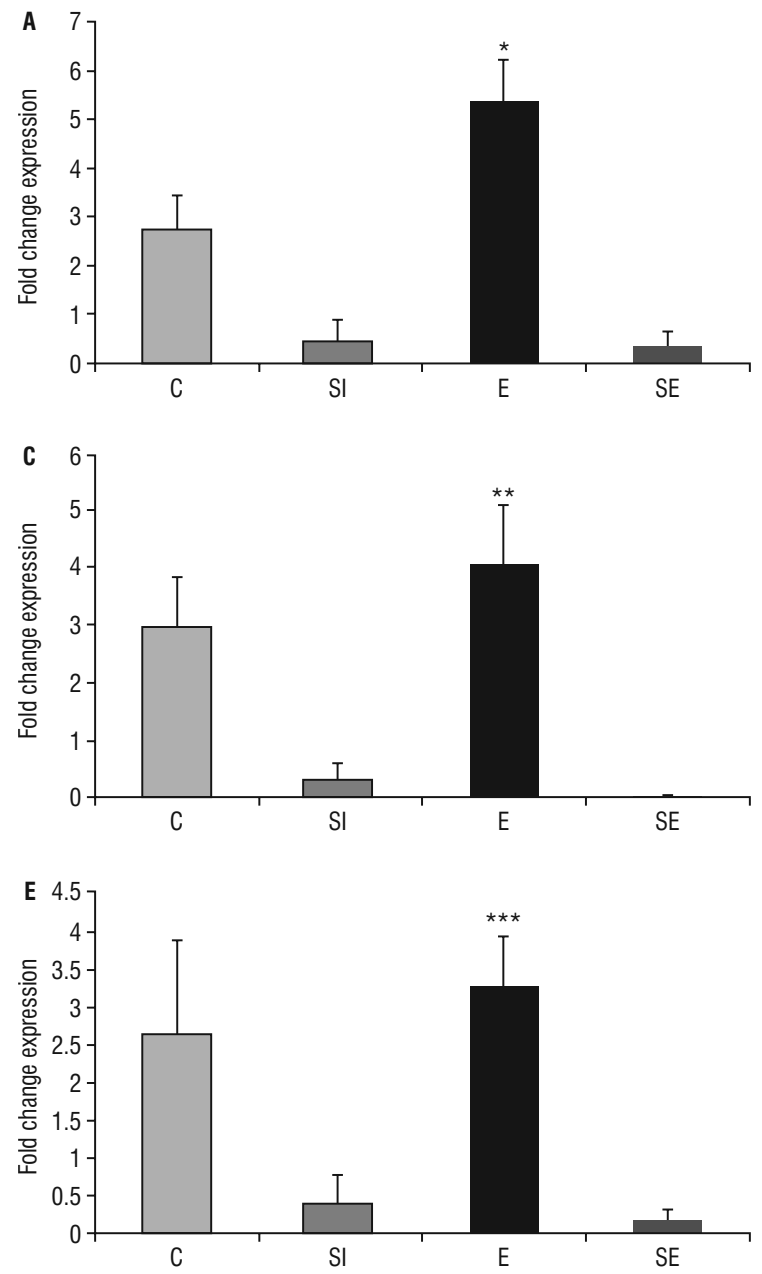

Together with NMDA receptor subunits gene expressions, we measured expression levels of BDNF, Cdk5, Cdk5r genes and found an increase in Cdk5 level in E group compare to the SE group significantly $(p=0.05)$. The ASCL1 gene expression increased in E group compare to the SE group $(p=0.031)$. The $D C X$ gene expression increasing in $C$ group compared to $S I$ and SE groups ( $p=0.20$ and $p=0.27)$. However, we did not find any significant differences in BDNF $(p=0.873), C d k 5 r(p=0.085), \operatorname{Arc}(p=0.46)$ and c-Fos gene level ( $p=0.079$; Fig. 2).

\section{DISCUSSION}

Generally, the performance for the novel object recognition declines while the interval between training and session increases, with better indices obtained around 90 min after training $[13,42]$. Our groups
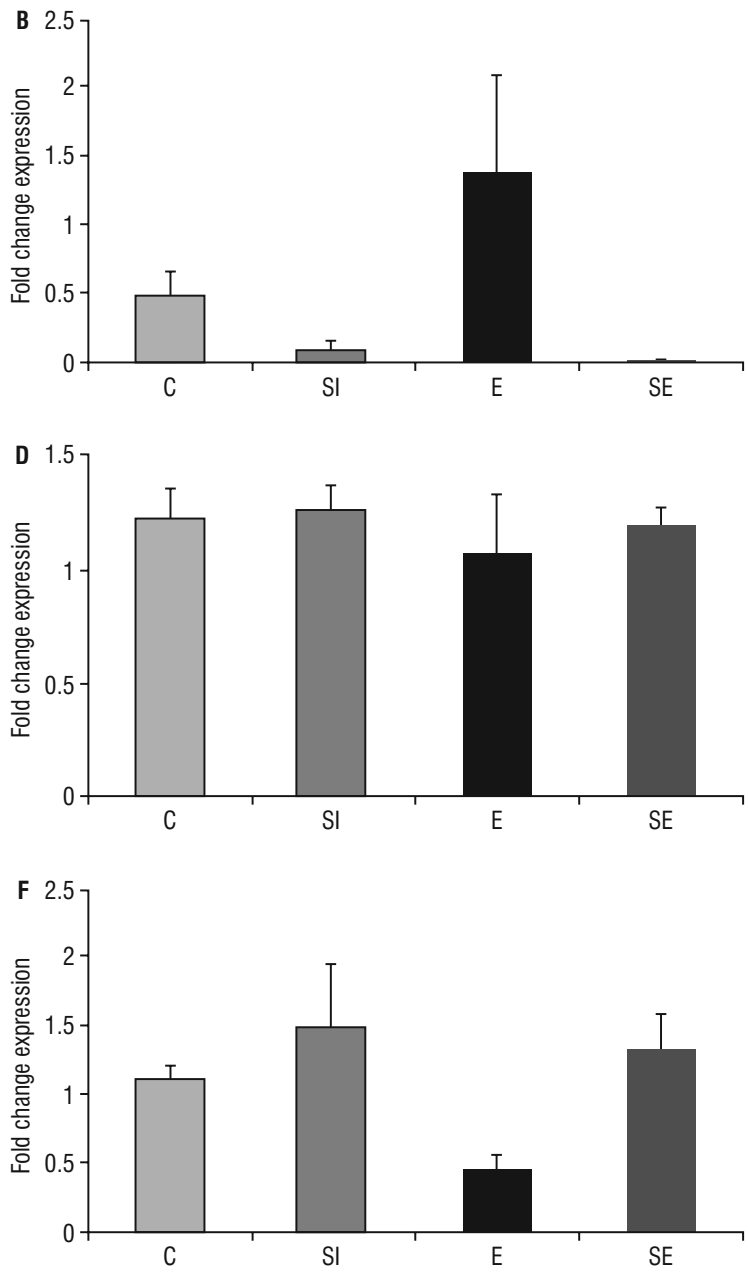

Figure 1. Shows fold change expression levels of Grin1 (A), Grin2a (B), Grin2b (C), brain-derived neurotrophic factor (BDNF; D), Cdk5 (E), $C d k 5 r(\mathbf{F})$, in rat hippocampus by real-time polymerase chain reaction. Values are expressed as a percentage of the cage control value $(100 \%)$. One way ANOVA followed by Tukey's post hoc multiple comparison tests; * for Grin1, different from others group (C, SI, SE); ${ }^{* *}$ for Grin2b, different from SI groups (SI and SE); ${ }^{* *}$ for Cdk5 different from SE group $(p<0.05) ; C$ - control group; $\mathrm{SI}$ - social isolation group; $\mathrm{E}$ - exercised group; SE - social isolation + exercise group. 

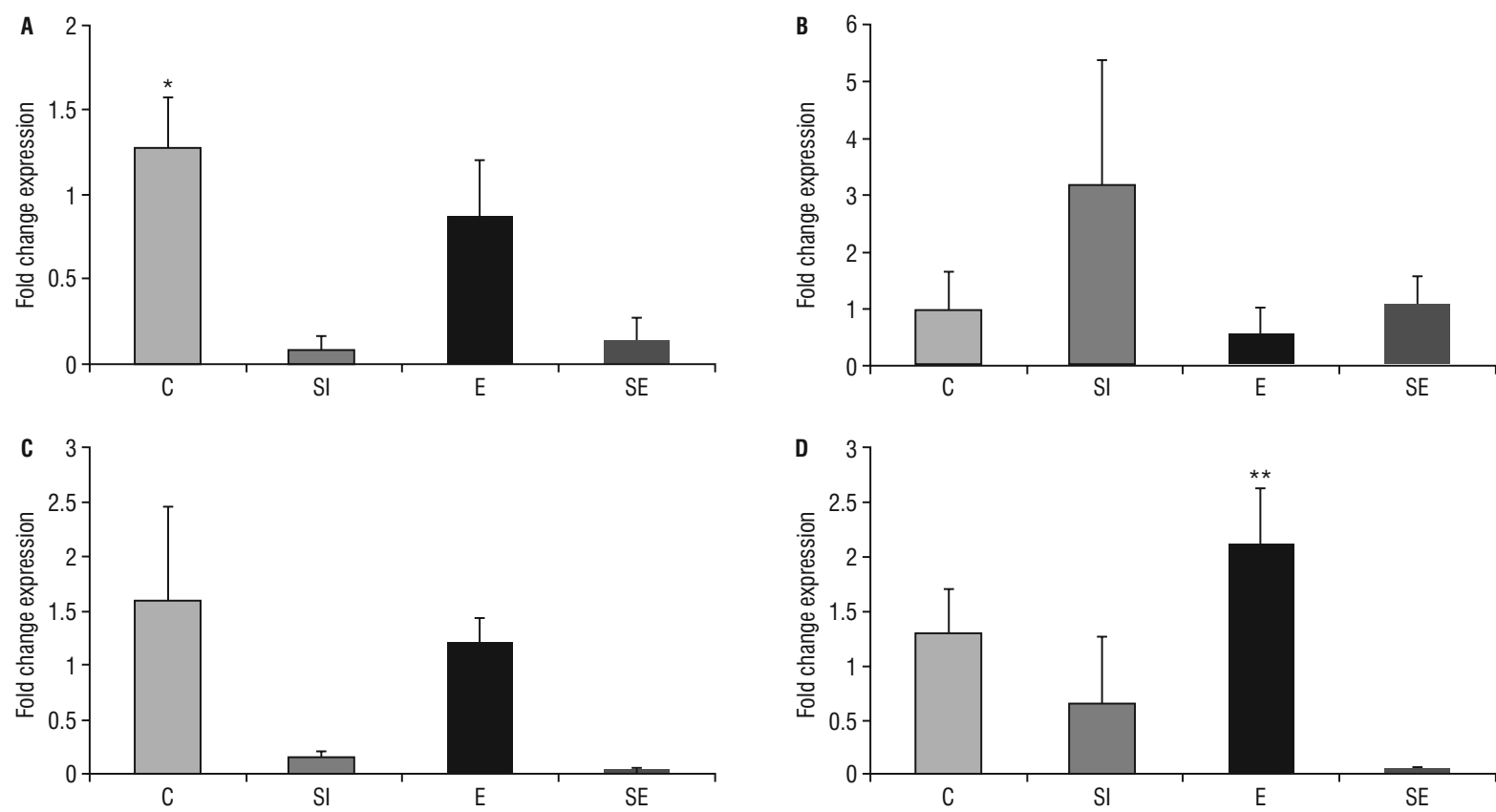

Figure 2. Shows fold change expression levels of doublecortin (DCX; A), Arc (B), C-Fos (C), achaete-scute homolog 1 (ASCL1; D) in rat hippocampus by real-time polymerase chain reaction. Values are expressed as a percentage of the cage control value (100\%). One way ANOVA followed by Tukey's post hoc multiple comparison tests; ${ }^{*}$ for DCX, SI and SE group; ${ }^{* *}$ for ASCL1, different from SE group $(p<0.05)$; $C-$ control group; $\mathrm{SI}$ - social isolation group; $\mathrm{E}$ - exercised group; $\mathrm{SE}$ - social isolation + exercise group.

showed no differences in the time spent in the novel object in $15 \mathrm{~min}$ and $240 \mathrm{~min}$ after training. This situation suggests that they did not recognise the novel object. In our study 60 min after training, social isolation group spent less time with the novel object compared to the control group. Exercise and social isolation + exercise rats did not show differences in the time spent in the novel object when compared to other groups, which means that both groups of rat effectively recognised the novel object. In our previous study, we found that social isolation stress and exercise did not affect the learning and memory functions by using the Morris water maze test. However, in the elevated plus maze test, anxiety scores were the lowest in the exercise group compared to others [6]. The novel object recognition test is based on the spontaneous novel object preference of rodents. Anatomically, this task is depending on the hippocampus, the nigrostriatal dopaminergic pathway and rhinal cortex [47]. These brain structures play a cardinal role in recognition memory formation, and if damage exists, the performance in recognition memory tasks is diminished [1]. It should be noted from that; another brain regions could affect the hippocampus process. Also in our study, long-term treadmill exercise did not show any possible effects on cognitive performances. Surprisingly, longer periods of exercise intervention are ineffective to promote cognitive performance compared to shorter durations. Possibly the induction of exercise in rodents is partly mediated by environmental changes [21]. Although we did not find differences in cognitive functions caused by exercise, we mentioned possible gene-environment interactions in the context of stress and exercise model. We showed an enhanced impact of exercise on learning and memory-related genes but not on some physiological measures.

Several psychiatric and neurological disorders manifest learning and memory deficits. For this reason, cognitive enhancement is considered as an effective treatment approach. NMDA is a main target gene for the development of cognitive enhancers due to its essential role in learning and memory. In particular, the NMDAR subunit gene NR2B improves synaptic plasticity and memory when over-expressed. However, the potential role as therapeutic target for NR2B gene has not been investigated detailed [5]. Here we showed in our study, Grin2b (NR2B), Grin1 (NR1) gene expressions increased in exercise group significantly. NMDAR subunits genes decreased in stress and exercise protocol, but this tendency was not significant. Like our results, Stranahan et al. [43] 
showed that the combined effect of stress and exercise diminished cognitive functions. Also Zhao et al. [50] indicated that NR2B gene was down-regulated in prefrontal cortex in response to isolation rearing for 8 weeks in Sprague Dawley rats. However, the fact that exercise seems to deficient treatment on social isolation stress should be taken into consideration. Because we showed social isolation + exercise groups' genes Grin2b, Grin1 and Grin 2a also downregulated like social isolation group. However, when exercise was applied to the grouping rats, it caused up-regulation in the Grin2b and Grin 1 genes expression levels. We can reveal that when experienced in a group setting, running stimulates learning and memory-related genes. Although NMDAR subunits genes altered due to the stress and exercise application, we did not find any changes in learning and memory. Possibly, duration of the exercise protocol was enough to express in genes' level but insufficient to reflect in learning and memory functions in rats. We presumed that, if we prolonged the exposure time of application we would probably see therapeutic effects of exercise on social isolation stress.

NMDAR subunit genes also interact with other signalling pathways such as Cdk5/p35. Cdk5 which is activated by binding to p35 or p39 is involved in synaptic plasticity and affects learning and memory formation [30]. In Cdk5 knockout (KO) mice and p35 KO mice, brain development is severely diminished because neuronal migration is impaired. Mishiba et al. [30] findings reveal that $\mathrm{Cdk5/p35}$ regulates spatial learning and memory, implicating Cdk5/p35 as a therapeutic target in neurological disorders. In addition, we showed that Cdk5 gene expression up-regulated in the exercise group. We have known from the literature that $\mathrm{Cdk} 5$ pathway has been identified closely related to NR2B in spatial learning and memory functions. According to our study, both NR2B and Cdk5 gene expression were up-regulated in the exercise group. To the best of our knowledge, we elaborated the effects of long-term treadmill exercise on the relationship between Cdk5 and NMDAR subunits firstly in literature.

Particularly acute stress leads to a decrease in BDNF level but declines in gene expression in chronic stress restricted $[31,48]$. Although our result cannot infer the relationship between BDNF and learning performances, we have known from the literature that learning activity can influence the BDNF level [7]. However, we are not sure if learning activity in the object recognition task could change immune content of these proteins [22]. In our study, BDNF level did not change between the group. Our forced exercise protocol did not affect BDNF levels. We assumed that when exercise applied voluntary, synaptic plasticity-related gene - BDNF could be altered [3]. However, in the modern era, people have not a tendency to do exercise voluntary and need induction for exercise in an obligated way. For this reason, forced treadmill exercise is suitable for people in their daily routine exercise. Nevertheless, we assumed that not just exercise type is important for people daily lives, also social interactions have really a cardinal role in learning and memory functions. These results suggest that in the absence of social interaction, a normally beneficial experience can exert a potentially deleterious influence on the brain. Nevertheless, it should be recalled that exercise protocol as itself can cause poor stress source and exaggerated diminished cognitive functions together with social isolation stress.

According to our results ASCL1, which involved in neural progenitor differentiation, was up-regulated in the exercise group significantly. Exercise has a clear impact on modifying microenvironment of progenitor immature cells. So here, we showed that new neurons are generated from stem cells usually in a quiescent state and they can reach to an active state by environmental stimulus like exercise. DCX expression is associated with neurite and axon elongation and synaptogenesis a marker of the new neuron [12]. In our study, we demonstrated that DCX down-regulated in stress and social isolation + exercise group compare to the control. Actual$l y$, the relationship between exercise and stress in modifying neurogenesis and cognitive processes in adulthood is complex. It has been demonstrated that social isolation inverted the positive effects of exercise in adulthood, followed a decrease in neuronal proliferation [43]. Our study suggested that, isolation stress during adolescence results in shrinks in neurogenesis in early adulthood rats. Thus, to our knowledge, this study is the first to demonstrate that longer-term exercise during adolescence does directly influences the expression levels of neurogenesis gene such as DCX, ASCL1 that reveal of new hippocampal neuron.

\section{CONCLUSIONS}

Long-term treadmill exercise increased hippocampal learning-related genes and neurogenesis without 
improving cognitive behaviour in socially isolated rats. Understanding the processes that underlie these changes should expand our knowledge of how environmental occasions may modify brain development and function, and development of neuropsychiatric disorders.

\section{Acknowledgements}

This study has been supported by Amasya University with the grand number, FMB-BAP-15-0160.

\section{REFERENCES}

1. Albasser MM, Davies M, Futter JE, et al. Magnitude of the object recognition deficit associated with perirhinal cortex damage in rats: Effects of varying the lesion extent and the duration of the sample period. Behav Neurosci. 2009; 123(1): 115-124, doi: 10.1037/a0013829, indexed in Pubmed: 19170436.

2. Barbour KA, Edenfield TM, Blumenthal JA. Exercise as a treatment for depression and other psychiatric disorders: a review. J Cardiopulm Rehabil Prev. 2007; 27(6): 359-367, doi: 10.1097/01.HCR.0000300262.69645.95, indexed in Pubmed: 18197069.

3. Bekinschtein P, Cammarota $M$, Katche $C$, et al. BDNF is essential to promote persistence of long-term memory storage. Proc Natl Acad Sci U S A. 2008; 105(7): 2711-2716, doi: 10.1073/pnas.0711863105, indexed in Pubmed: 18263738.

4. Bekinschtein P, Oomen CA, Saksida LM, et al. Effects of environmental enrichment and voluntary exercise on neurogenesis, learning and memory, and pattern separation: BDNF as a critical variable? Semin Cell Dev Biol. 2011; 22(5): 536-542, doi: 10.1016/j.semcdb.2011.07.002, indexed in Pubmed: 21767656.

5. Benite-Ribeiro SA, Santos JM, Duarte JA. Moderate physical exercise attenuates the alterations of feeding behaviour induced by social stress in female rats. Cell Biochem Funct. 2014; 32(2): 142-149, doi: 10.1002/cbf.2984, indexed in Pubmed: 23740556.

6. Cevik OS, Sahin L, Tamer L. Long term treadmill exercise performed to chronic social isolated rats regulate anxiety behavior without improving learning. Life Sci. 2018; 200: 126-133, doi: 10.1016/j.Ifs.2018.03.029, indexed in Pubmed: 29559338.

7. Chen J, Kitanishi T, Ikeda T, et al. Contextual learning induces an increase in the number of hippocampal CA1 neurons expressing high levels of BDNF. Neurobiol Learn Mem. 2007; 88(4): 409-415, doi: 10.1016/j.nlm.2007.07.009, indexed in Pubmed: 17765576.

8. Chen WG, Chang Q, Lin Y, et al. Derepression of BDNF transcription involves calcium-dependent phosphorylation of MeCP2. Science. 2003; 302(5646): 885-889, doi: 10.1126/ science.1086446, indexed in Pubmed: 14593183.

9. Contreras-Vallejos E, Utreras E, Bórquez DA, et al. Searching for novel Cdk5 substrates in brain by comparative phosphoproteomics of wild type and Cdk5-/- mice. PLoS One. 2014; 9(3): e90363, doi: 10.1371/journal. pone.0090363, indexed in Pubmed: 24658276.

10. Cotman CW, Berchtold NC, Christie LA. Exercise builds brain health: key roles of growth factor cascades and inflammation. Trends Neurosci. 2007; 30(9): 464-472, doi: 10.1016/j.tins.2007.06.011, indexed in Pubmed: 17765329.
11. Cull-Candy SG, Leszkiewicz DN. Role of distinct NMDA receptor subtypes at central synapses. Sci STKE. 2004; 2004(255): re16, doi: 10.1126/stke.2552004re16, indexed in Pubmed: 15494561.

12. D'Alessio L, Konopka H, López EM, et al. Doublecortin (DCX) immunoreactivity in hippocampus of chronic refractory temporal lobe epilepsy patients with hippocampal sclerosis. Seizure. 2010; 19(9): 567-572, doi: 10.1016/j. seizure.2010.09.004, indexed in Pubmed: 20888264.

13. de Bruin N, Pouzet B. Beneficial effects of galantamine on performance in the object recognition task in Swiss mice: deficits induced by scopolamine and by prolonging the retention interval. Pharmacol Biochem Behav. 2006; 85(1): 253-260, doi: 10.1016/j.pbb.2006.08.007, indexed in Pubmed: 16987546.

14. Ennaceur A, Meliani K. A new one-trial test for neurobiological studies of memory in rats. III. Spatial vs. non-spatial working memory. Behav Brain Res. 1992; 51(1): 83-92, doi: 10.1016/s0166-4328(05)80315-8.

15. Fone KCF, Porkess MV. Behavioural and neurochemical effects of post-weaning social isolation in rodents-relevance to developmental neuropsychiatric disorders. Neurosci Biobehav Rev. 2008; 32(6): 1087-1102, doi: 10.1016/j. neubiorev.2008.03.003, indexed in Pubmed: 18423591.

16. Francis F, Koulakoff A, Boucher D, et al. Doublecortin is a developmentally regulated, microtubule-associated protein expressed in migrating and differentiating neurons. Neuron. 1999; 23(2): 247-256, doi: 10.1016/ s0896-6273(00)80777-1, indexed in Pubmed: 10399932.

17. Gleeson J, Lin P, Flanagan L, et al. Doublecortin is a microtubule-associated protein and is expressed widely by migrating neurons. Neuron. 1999; 23(2): 257-271, doi: 10.1016/s0896-6273(00)80778-3.

18. Green MR, McCormick CM. Effects of stressors in adolescence on learning and memory in rodent models. Horm Behav. 2013; 64(2): 364-379, doi: 10.1016/j.yhbeh.2012.09.012, indexed in Pubmed: 23998678.

19. Gubern $C$, Hurtado $O$, Rodríguez $R$, et al. Validation of housekeeping genes for quantitative real-time PCR in in-vivo and in-vitro models of cerebral ischaemia. BMC Mol Biol. 2009; 10: 57, doi: 10.1186/1471-2199-10-57, indexed in Pubmed: 19531214.

20. Hamilton GF, Rhodes JS. Exercise regulation of cognitive function and neuroplasticity in the healthy and diseased brain. Prog Mol Biol Transl Sci. 2015; 135: 381-406, doi: 10.1016/bs.pmbts.2015.07.004, indexed in Pubmed: 26477923.

21. Hatchard T, Ting JJ, Messier C. Translating the impact of exercise on cognition: methodological issues in animal research. Behav Brain Res. 2014; 273: 177-188, doi: 10.1016/j. bbr.2014.06.043, indexed in Pubmed: 25026095.

22. Heldt SA, Stanek L, Chhatwal JP, et al. Hippocampus-specific deletion of BDNF in adult mice impairs spatial memory and extinction of aversive memories. Mol Psychiatry. 2007; 12(7): 656-670, doi: 10.1038/sj.mp.4001957, indexed in Pubmed: 17264839.

23. Hong S, Flashner B, Chiu M, et al. Social isolation in adolescence alters behaviors in the forced swim and sucrose preference tests in female but not in male rats. Physiol Behav. 2012; 105(2): 269-275, doi: 10.1016/j. physbeh.2011.08.036, indexed in Pubmed: 21907226.

24. Hong YP, Lee HC, Kim HT. Treadmill exercise after social isolation increases the levels of NGF, BDNF, and synapsin $I$ to induce survival of neurons in the hippocampus, and improves depression-like behavior. J Exerc Nutrition Biochem. 2015; 19: 11-18, doi: 10.5717/jenb.2015.19.1.11. 
25. Imayoshi I, Kageyama R. bHLH factors in self-renewal, multipotency, and fate choice of neural progenitor cells. Neuron. 2014; 82(1): 9-23, doi: 10.1016/j.neuron.2014.03.018, indexed in Pubmed: 24698265.

26. Kuruba R, Hattiangady B, Shetty AK. Hippocampal neurogenesis and neural stem cells in temporal lobe epilepsy. Epilepsy Behav. 2009; 14 Suppl 1: 65-73, doi: 10.1016/j. yebeh.2008.08.020, indexed in Pubmed: 18796338.

27. Lander SS, Linder-Shacham D, Gaisler-Salomon I. Differential effects of social isolation in adolescent and adult mice on behavior and cortical gene expression. Behav Brain Res. 2017; 316: 245-254, doi: 10.1016/j.bbr.2016.09.005, indexed in Pubmed: 27618762.

28. Li S, Luo J, Wang Xi, et al. Effects of Ginkgo biloba extracts on NMDA-activated currents in acutely isolated hippocampal neurons of the rat. Phytother Res. 2011; 25(1): 137-141, doi: 10.1002/ptr.3235, indexed in Pubmed: 20632296.

29. McCormick CM, Thomas CM, Sheridan CS, et al. Social instability stress in adolescent male rats alters hippocampal neurogenesis and produces deficits in spatial location memory in adulthood. Hippocampus. 2012; 22(6): 1300-1312, doi: 10.1002/hipo.20966, indexed in Pubmed: 21805526.

30. Mishiba T, Tanaka M, Mita N, et al. Cdk5/p35 functions as a crucial regulator of spatial learning and memory. Mol Brain. 2014; 7: 82, doi: 10.1186/s13041-014-0082-x, indexed in Pubmed: 25404232.

31. Murakami S, Imbe H, Morikawa Y, et al. Chronic stress, as well as acute stress, reduces BDNF mRNA expression in the rat hippocampus but less robustly. Neurosci Res. 2005; 53(2): 129-139, doi: 10.1016/j.neures.2005.06.008, indexed in Pubmed: 16024125.

32. Paoletti P, Bellone C, Zhou Q. NMDA receptor subunit diversity: impact on receptor properties, synaptic plasticity and disease. Nat Rev Neurosci. 2013; 14(6): 383-400, doi: 10.1038/nrn3504, indexed in Pubmed: 23686171.

33. Papa FT, Mancardi MM, Frullanti E, et al. Personalized therapy in a GRIN1 mutated girl with intellectual disability and epilepsy. Clin Dysmorphol. 2018; 27(1): 18-20, doi: 10.1097/MCD.0000000000000205, indexed in Pubmed: 29194067.

34. Pleasure SJ, Collins AE, Lowenstein DH. Unique expression patterns of cell fate molecules delineate sequential stages of dentate gyrus development. J Neurosci. 2000; 20(16): 6095-6105, doi: 10.1523/JNEUROSCI.20-16-06095.2000, indexed in Pubmed: 10934259.

35. Peebles CL, Yoo J, Thwin MT, et al. Arc regulates spine morphology and maintains network stability in vivo. Proc Natl Acad Sci U S A. 2010; 107(42): 18173-18178, doi: 10.1073/ pnas.1006546107, indexed in Pubmed: 20921410.

36. Plath N, Ohana O, Dammermann B, et al. Arc/Arg3.1 is essential for the consolidation of synaptic plasticity and memories. Neuron. 2006; 52(3): 437-444, doi: 10.1016/j. neuron.2006.08.024, indexed in Pubmed: 17088210.

37. Plattner F, Hernández A, Kistler TM, et al. Memory enhancement by targeting Cdk5 regulation of NR2B. Neuron. 2014; 81(5): 1070-1083, doi: 10.1016/j.neuron.2014.01.022, indexed in Pubmed: 24607229.

38. Quan MN, Tian YT, Xu KH, et al. Post weaning social isolation influences spatial cognition, prefrontal cortical synaptic plasticity and hippocampal potassium ion chan- nels in Wistar rats. Neuroscience. 2010; 169(1): 214-222, doi: $10.1016 / j$.neuroscience.2010.04.048, indexed in Pubmed: 20438813.

39. Raineki C, Cortés MR, Belnoue L, et al. Effects of early-life abuse differ across development: infant social behavior deficits are followed by adolescent depressive-like behaviors mediated by the amygdala. J Neurosci. 2012; 32(22): 7758-7765, doi: 10.1523/JNEUROSCI.5843-11.2012, indexed in Pubmed: 22649253.

40. Sandstrom NJ, Hart SR. Isolation stress during the third postnatal week alters radial arm maze performance and corticosterone levels in adulthood. Brain. Res. 2005; 156(22): 289-296.

41. Schöfl C, Waring M, Bergwitz C, et al. Cyclic-adenosine 3', 5'-monophosphate-stimulated c-fos gene transcription involves distinct calcium pathways in single beta-cells. Mol Cell Endocrinol. 2002; 186(1): 121-131, doi: 10.1016/ s0303-7207(01)00609-8, indexed in Pubmed: 11850128.

42. Sik A, van Nieuwehuyzen P, Prickaerts J, et al. Performance of different mouse strains in an object recognition task. Behav Brain Res. 2003; 147(1-2): 49-54, indexed in Pubmed: 14659569.

43. Stranahan AM, Khalil D, Gould E. Social isolation delays the positive effects of running on adult neurogenesis. Nat Neurosci. 2006; 9(4): 526-533, doi: 10.1038/nn1668, indexed in Pubmed: 16531997.

44. Tao X, Finkbeiner S, Arnold DB, et al. Ca2+ influx regulates BDNF transcription by a CREB family transcription factor-dependent mechanism. Neuron. 1998; 20(4): 709-726, doi: 10.1016/s0896-6273(00)81010-7, indexed in Pubmed: 9581763.

45. Taylor H, Jaimee TJ, Claude M. Translating the impact of exercise on cognition: methodological issues in animal research. Behav Brain Res. 2014; 273: 177-188, doi: 10.1016/j.bbr.2014.06.043, indexed in Pubmed: 25026095.

46. van den Buuse M, Ruimschotel E, Martin S, et al. Enhanced effects of amphetamine but reduced effects of the hallucinogen, 5-MeO-DMT, on locomotor activity in 5-HT(1A) receptor knockout mice: implications for schizophrenia. Neuropharmacology. 2011; 61(1-2): 209-216, doi: 10.1016/j.neuropharm.2011.04.001, indexed in Pubmed: 21501627.

47. Winters BD, Bussey TJ. Glutamate receptors in perirhinal cortex mediate encoding, retrieval, and consolidation of object recognition memory. J Neurosci. 2005; 25(17): 4243-4251, doi: 10.1523/JNEUROSCI.0480-05.2005, indexed in Pubmed: 15858050.

48. Xu H, Luo C, Richardson JS, et al. Recovery of hippocampal cell proliferation and BDNF levels, both of which are reduced by repeated restraint stress, is accelerated by chronic venlafaxine. Pharmacogenomics J. 2004; 4: 322-331.

49. Yuzaki M. Cerebellar LTD vs. motor learning-lessons learned from studying GluD2. Neural Netw. 2013; 47: 36-41, doi: 10.1016/j.neunet.2012.07.001, indexed in Pubmed: 22840919.

50. Zhao X, Sun L, Jia $H$, et al. Isolation rearing induces social and emotional function abnormalities and alters glutamate and neurodevelopment-related gene expression in rats. Prog Neuropsychopharmacol Biol Psychiatry. 2009; 33(7): 1173-1177, doi: 10.1016/j.pnpbp.2009.06.016, indexed in Pubmed: 19563853. 